\title{
Using Arduino microcontroller boards to measure response latencies
}

\author{
Thomas W. Schubert • Alessandro D'Ausilio • \\ Rosario Canto
}

Published online: 13 April 2013

(C) Psychonomic Society, Inc. 2013

\begin{abstract}
Latencies of buttonpresses are a staple of cognitive science paradigms. Often keyboards are employed to collect buttonpresses, but their imprecision and variability decreases test power and increases the risk of false positives. Response boxes and data acquisition cards are precise, but expensive and inflexible, alternatives. We propose using open-source Arduino microcontroller boards as an inexpensive and flexible alternative. These boards connect to standard experimental software using a USB connection and a virtual serial port, or by emulating a keyboard. In our solution, an Arduino measures response latencies after being signaled the start of a trial, and communicates the latency and response back to the PC over a USB connection. We demonstrated the reliability, robustness, and precision of this communication in six studies. Test measures confirmed that the error added to the
\end{abstract}

Electronic supplementary material The online version of this article (doi:10.3758/s13428-013-0336-z) contains supplementary material, which is available to authorized users.

T. W. Schubert

Instituto Universitário de Lisboa (ISCTE-IUL),

Centro de Investigação e Intervenção Social,

Av. Forças Armadas, Ed. ISCTE-IUL,

1649-026 Lisbon, Portugal

A. D'Ausilio · R. Canto

RBCS-Robotics, Brain and Cognitive Sciences Department, Italian Institute of Technology (IIT), Genoa, Italy

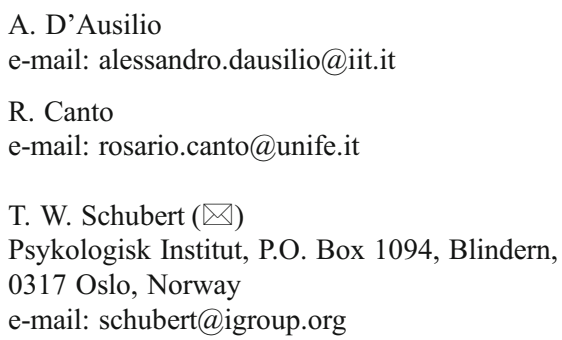

measurement had an $S D$ of less than $1 \mathrm{~ms}$. Alternatively, emulation of a keyboard results in similarly precise measurement. The Arduino performs as well as a serial response box, and better than a keyboard. In addition, our setup allows for the flexible integration of other sensors, and even actuators, to extend the cognitive science toolbox.

Keywords Response latencies $\cdot$ Reaction times $\cdot$ Response box $\cdot$ Arduino $\cdot$ E-Prime

Paradigms that measure latencies of responses are a staple of many areas of experimental research on cognition. In theory, latencies of many different actions can be measured: keypresses, spoken answers, or movements of fingers, hands, eyes, or other body parts.

Very often, however, experimental psychologists simply ask participants to react to computer-presented stimuli by pressing keys on regular keyboards. We believe this is unfortunate for two reasons: First, regular keyboards are notoriously imprecise input devices that add noise to the data. Second, keypresses are most often arbitrarily matched to the outcome of some cognitive process, whereas other movements might index the investigated process more directly. Experimental psychologists may be aware of both shortcomings and still choose the keyboard out of habit, convenience, or lack of alternatives.

With the present work, we propose an alternative platform that allows both more precise measurement and more creative dependent variables. It uses the open-source Arduino microcontroller platform and thereby harvests the creativity of a large developer community. In the following, we will elaborate on the imprecision of regular keyboards, before we describe and test how the Arduino can be used as a response latency measurement platform. 


\section{Keyboards and response boxes}

Before we discuss the quality of response time measurement, we need to define the terms for doing so. Following Plant and Turner (2009), we will use the terms accuracy and precision to denote two different concepts. A response latency measurement is accurate when it is close to the actual time a response took. Imagine that you measure how long it takes a $1-\mathrm{kg}$ ball with a radius of $10 \mathrm{~cm}$ to roll down a $30^{\circ}$ slope for $1 \mathrm{~m}$, repeating it many times (Galilei, 1638). If the average time is $903 \mathrm{~ms}$, the measurement is accurate. Most likely your measures will be distributed normally around the average. The narrower the distribution, the more precise we call the measurement. It is possible to have a very precise measurement (a very low standard deviation) around an inaccurate average. This would be unfortunate for determining $g$ from your measures, but it is typical for measures of response latencies in cognitive science when the focus is on differences between conditions, and less on the absolute size (within certain limits). Note however that there are situations where accuracy is more important, for instance when ratios instead of differences between latencies are investigated. We will focus on precision in this article, but also reflect on accuracy when we have the numbers.

Even when using special software that synchronizes stimulus onset with the refresh cycle of the monitor, regular keyboards do not allow millisecond precise measuring of response latencies. Instead, the measured response latencies distribute normally around the true latency with a standard deviation that ranges from 1 to several milliseconds depending on the hardware (Voss, Leonhart, \& Stahl, 2007).

For instance, Plant and Turner (2009) $)^{1}$ found that a USB keyboard (Belkin) added an error with an average $M=$ $18.30 \mathrm{~ms}$ and a standard deviation $S D=1.29 \mathrm{~ms}$. Two other keyboards connected by serial port added different errors to the latencies, with $M=33.73, S D=3.08$, and $M=19.94$, $S D=0.083$. They also tested four computer mice connected by USB, and reported errors averages between 18 and $49 \mathrm{~ms}$, and $S D$ s between 0.7 and $4.28 \mathrm{~ms}$. K. I. Forster and Forster (2003; see also Forster, 2012) tested one keyboard that produced measured latencies with a standard deviation of $5.15 \mathrm{~ms}$; several USB mice ranged from $S D=$ 5.65 to $S D=6.74$, and two gamepads produced lower $S D$ s (1.33 and 2.61). It is easy to imagine that with such a range of different distributions, using different equipment in a lab can quickly lead to erroneous results, and even sticking to one type of hardware adds noise to the data.

To overcome such imprecision, experimenters can employ special hardware that allows more precise measurement, for

\footnotetext{
${ }^{1}$ For a setup using Windows XP SP2 and E-Prime 1.3, as well as the Black Box ToolKit (www.blackboxtoolkit.com), see Plant, Hammond, and Turner (2004).
}

instance so-called response boxes. These response boxes provide a few keys in a special and fixed layout, and typically promise millisecond-precise measurement of latencies. Software for experimentation assures synchronization with the stimulus display (visual or audio) and records the response and its latency. Response boxes are connected to the computer either using a serial port, or via USB, depending on the model.

Increased precision of measurement provided by response boxes should translate into obvious methodological advances: Test power increases, and thus fewer participants and/or trials are needed to detect an effect, or smaller effects can be detected. At the same time, the risk of rejecting the null hypothesis because of a spurious result arising by chance should decrease.

Nevertheless, the use of response boxes is by no means universal. In many cases, this may be due to the robustness of typical paradigms: Some paradigms produce effects large enough to make the noise introduced by keyboards negligible. In other cases, however, researchers may be motivated to use response boxes, but do not because of the cost of the hardware (especially when running many participants in parallel) or technical difficulties in setting them up. Often, commercial response boxes only function (well) in concert with one particular software.

Viable alternatives to response boxes exist, but share some of the disadvantages. For instance, response boxes can be fashioned out of cheap equipment such as computer mice, and connected to the parallel port on a PC (Voss et al., 2007). However, parallel ports are disappearing fast from modern computers, and are not available on modern laptops, although alternative USB solutions have been proposed (Canto, Bufalari, \& D'Ausilio, 2011). Also, multipurpose hardware can be used to create response box functionality: Data Acquisition Cards are microprocessor boards that are plugged into expansion slots of desktop PCs, and allow digital input and output. They can be connected to external buttons and measure the time at which those buttons are pressed (Harmon-Jones, 2011). For instance, this is currently the only way to provide millisecond-precise response latency measurement in the free software package DMDX (Forster \& Forster, 2003).

Empirical reports confirm the accuracy and precision of measurement that uses response boxes with serial connections, data acquisition cards, or parallel ports: The error added has typically a rather low average (around $6 \mathrm{~ms}$ ), and a low standard deviation - in most cases less than or close to $1 \mathrm{~ms}$ (Forster, 2012; Plant, Hammond, \& Whitehouse, 2003; Voss et al., 2007). Unfortunately, we know of no empirical report on the precision of USBconnected response boxes except the work by $\mathrm{Li}$, Liang, Kleiner, and Lu (2010) on their own device. They showed a remarkable precision below $1 \mathrm{~ms}$ in concert with MATLAB and the Psychophysics Toolbox software (Brainard, 1997). 
An obvious downside to response boxes is that they measure only presses (and releases) of buttons. While it would be unfair to caricature cognitive science as the study of beings with only one eye and two fingers, there is reason to believe that cognitive science overly relies on investigating choice decisions enacted with pressing one of two buttons. Unfortunately, measuring the latencies of moving fingers, hands, heads, or feet requires special hardware that costs even more, is even more difficult to set up than response boxes, and often only used by large labs that have access to a specialized technician.

To sum up: Response boxes provide much more precise measurement of the latency of button presses; yet, they are often not employed, and inherently restricted because of their specialized nature.

\section{The present research}

In this article, we propose to use the Arduino microprocessor platform as an alternative to keyboards and standard response boxes. We show how to connect an Arduino to standard reaction time software (using E-Prime), and report how precise the measurement is compared to standard response boxes. We also sketch how using the Arduino platform allows extending the experimental toolbox to include other measures beyond key presses. Thus, we describe how the Arduino can be used as a platform for measuring latencies of various kinds.

Our empirical strategy was first to evaluate how best to communicate between experimental software on a PC and an attached Arduino in Study 1, and then to develop scripts for E-Prime and Arduino that implement this communication. The robustness of these scripts was evaluated in Study 2. In Study 3, we tested the speed of our platform by having it detect onset of stimuli on a screen. Study 4 was designed to compare the speed of the platform to a serial response box and a standard keyboard by using a "robot" that detects stimuli onsets and hits buttons with a solenoid. In Study 5, we took a closer look at increasing the accuracy of latency measurement. Finally, we performed Study 6 to investigate the performance of a simpler version of communication that could be used with any experimental software, not just E-Prime.

\section{The Arduino platform}

Arduino is the name of a family of microcontroller boards. The boards are a combination of an ATMEL microprocessor including RAM, flash memory, and input/output channels. Thus, these boards have the same general structure as common personal computers, but their performance is of course only a fraction of those. In the present article, we mainly use the recent reference model of the Arduino family, the Arduino Uno. It has $32 \mathrm{kB}$ of flash memory, operates at $16 \mathrm{MHz}$, and features 14 digital input/output and 6 analog input pins. (Other, larger models provide more of those input and output options.) Part of the Arduino package is a programming environment, where code is written in a simplified C-like language, and transferred to the Arduino using a USB cable. After programming, an Arduino can work while being connected to a PC (and thereby transmit data), or operate standalone.

Several features make the Arduino family an interesting tool as a measurement platform. First of all, it connects easily by USB to a Windows PC, Mac, or Linux machine, and can transmit data using a virtual serial port to these operating systems. Second, it is open source hardware, which means that everybody can access, modify, and use the board design. Likewise, the software is free and open source. This has led to both a low price of a board (around $€ 20-30$, or \$35) and a large community that develops hardware and software compatible with the Arduino.

The boards can be connected to actuators and sensors, either commercial or build from scratch. Many extensions of a board come as so-called shields, additional small boards that are plugged into an Arduino board. For instance, some shields provide wireless access (WLAN or Bluetooth), whereas others allow storing data on flash memory cards. In addition, this idea of extending a board by plugging in components is taken further by rapid prototyping platforms, where smaller components are connected by plugging them into special connection shields. For instance, the prototyping platforms Tinkerkit and Seeedstudio Grove enable users to connect LEDs, small vibrating motors, buttons, accelerometers, gyroscopes, or hall sensors to the Arduino within min. Together with the active community and freely available code, this guarantees a wide range of possibilities to create new kinds of measurements.

Despite its simplicity and low price, the Arduino can function as a reliable controller for experimental settings. When investigating how an Arduino can replace more complicated hardware as a standalone controller of experimental input and stimuli, D'Ausilio (2012) confirmed that signals generated by an Arduino were reliably constant in length and delay. He also found that typical combinations of input and output were performed with remarkable accuracy and precision, often with standard deviations of only microseconds. However, he did not yet explore how to embed Arduinos into response latency measures run by a PC.

Integrating Arduino into PC-controlled experimental setups

With its speed and flexibility, Arduino boards could serve as useful platforms to build response devices that allow experimental setups with buttons, other sensors, and even output 
hardware. The challenge is to connect the Arduino to experimental software in a way that allows fast and reliable measurement.

In principle, there are several options to accomplish communication between an Arduino board and the computer that is executing the experiment. Here, we opt for a method that does not rely on additional or legacy hardware, namely to simply use the USB connection available on modern Arduino boards. ${ }^{2}$ Most Arduino boards are outfitted with additional chips that convert the serial communication from the microprocessor into USB that connects then to a PC (these converter chips are Atmega16U2 on the Arduino Uno R3, Atmega8U2 on earlier models, or FTDI serial-toUSB chips in older boards). Some Arduino boards come without such a connector, and it can then be added temporarily for programming. The more recent Arduino Leonardo board uses the Atmel ATmega32u4 microprocessor that has built-in USB communication and thus does not require an additional converter chip.

Irrespective of the hardware, the result is that a USB cable connects Arduino and PC, and provides a serial connection. On the PC side, driver software creates a virtual serial (COM) port. This serial port can be accessed with any software that can communicate with a serial port. The driver offers several baud rates; for instance, the driver for the Uno offers 57,600 and 115,200 baud as the fastest speeds. Note that other boards will offer other baud rates, including 128,000 baud.

Apart from choosing USB as the access hardware, there are at least two possible software strategies to access experimental hardware through the Arduino. One approach would be to use the Arduino as a relatively "dumb" gateway, another one would be to use its capabilities more fully, and in particular to have it measure latencies itself. Let us outline these approaches in more detail. As an example, imagine that two buttons are connected to the Arduino that are used as response keys in a reaction time study.

Treating the Arduino as a dumb gateway, its programming would simply continuously read the states of the two input pins to which the buttons are connected. When one of the buttons is pressed, this input is detected by the Arduino. It is programmed to then send a signal via the serial interface, which could simply consist of one character indicating which button was pressed. The connected PC, running the experiment, would present a stimulus and then listen on the

\footnotetext{
$\overline{{ }^{2} \text { We can think }}$ of three alternatives: First, Arduino boards can be outfitted with serial connectors, which would allow connecting them to a PC with a serial input. Second, Arduino output pins can be connected to a data acquisition card's input pins or to a parallel port. Both would result in fast and flexible solutions, but also require legacy or additional hardware that we want to replace. A third solution would establish a wireless connection via WLAN or Bluetooth; however, time lags added to the transmissions would have to be evaluated carefully.
}

serial port for the response to arrive (or until a timeout is reached).

Treating the Arduino as a microcomputer with timing capabilities, the procedure could take a different, second form. In this case, the PC would signal the Arduino via the serial port when a response is expected (e.g., after presenting a stimulus to which the participant has to respond). When receiving this signal, the Arduino saves a time stamp, and starts waiting for the response. When the response is detected, the Arduino again takes a time stamp. It then transmits the difference as the response latency, and which response was given. This is the approach taken by the RTBox response box (Li et al., 2010).

The two approaches differ in several aspects. The first approach requires less programming. However, it is also less flexible; for instance, it would not afford the presentation of some stimuli or feedback via the Arduino itself. The two approaches also differ regarding robustness against lags in the serial connection. The first solution would only suffer from lag in serial communication from the Arduino to the $\mathrm{PC}$, whereas the second solution would suffer only from lag in the serial communication from PC to Arduino. Study 1 was designed to investigate the lags of these two directions in order to decide which strategy is appropriate.

\section{Study 1: Lags in USB communication between PC and Arduino}

For this test, we connected an Arduino board via USB to a PC running E-Prime 2. Both the PC and the Arduino board were also connected to another $\mathrm{PC}$ with a data acquisition (DAQ) card equipped with a dedicated hardware clock. We had E-Prime send data to the Arduino, and the Arduino sent it then back. With the external clock in the DAQ card, we measured the duration of each leg.

\section{Method}

We used an Arduino UNO R1, and a PC (Intel Core 2 Quad Q6600, 2.4 Ghz) running Windows 7 and E-Prime 2.0.10.182. Serial port connections were set to 128,000 baud. The external DAQ was a CED Micro $1401 \mathrm{mk}$ II (from Cambridge Electronics Devices, UK) sampling at $10000 \mathrm{~Hz}$.

In each test trial, the sequence of events was as follows: (1) the E-Prime script sent an initial TTL pulse to the external timer to indicate the start of the trial. E-Prime then sent an integer (two bytes) over the serial port to the connected Arduino. As soon as the Arduino received two bytes, it set one of its output lines to HIGH, thereby signaling the external clock connected to this line that it received data. It then waited for $300 \mathrm{~ms}$ (similar to a minimal 
response latency), and then sent the received integer back to the serial port. As soon as E-Prime received the integer, it again sent a TTL to the external clock, signaling the end of the round trip. We ran 990 of such trials.

\section{Results}

For the leg from E-Prime to Arduino, we measured durations that varied between 1.2 and $1.5 \mathrm{~ms} ; M=1.251 \mathrm{~ms}, S D=$ $0.051 \mathrm{~ms}$. For the leg from Arduino back to E-Prime, durations varied between 1.9 and $4.9 \mathrm{~ms}, M=3.080 \mathrm{~ms}, S D=$ $0.417 \mathrm{~ms}$. In all cases, the received integer was the one that was sent.

These data suggest that sending data to the Arduino provides less absolute delay, but more importantly also a lot less variability of the delay. Thus, the more promising strategy is to (1) signal the Arduino the beginning of a trial, (2) have it measure the reaction time itself, and then (3) send the measured latencies back. This approach will be followed now, but we come back to the first approach in Study 6 .

\section{Connecting an Arduino response box to E-Prime}

\section{Scripting E-Prime to interface Arduino}

In the following section, we will demonstrate how an Arduino can be integrated into a common software for running experiments, namely E-Prime. E-Prime has a built-in interface to serial ports. It does not, however, treat the serial port as a built-in input device. To integrate the Arduino, we thus use a script (called InLine in E-Prime) that communicates with the Arduino, and updates the response logs accordingly.

As a first step, a serial device needs to be added to the Devices tab of the experiment. We choose the name serial, 115,200 bits per second, eight data bits, no parity, and one stop bit as default values. The COM port and its specifications have to correspond to those assigned to the virtual serial port of the Arduino (which can be checked in Windows Device Manager, where also the baud rate can be adjusted to the one used in E-Prime).

Adding fields to the stimulus table We then set up stimulus presentations inside a list, as it is commonly done in EPrime. Our list for the stimuli is called StimulusList, and for the stimulus presentation we use a TextDisplay Object named trialStimulus. In the StimulusList table, we add several additional properties: TrialDuration, which is transmitted to the Arduino and tells it the maximum time in milliseconds to wait for a response; this is the only additional field that the experimenter has to fill. Next, we add TrialN $r$, which will contain a random trial identifier number.
We also add ArduinoTrialNr, ArduinoRT, ArduinoButtons, which are the three values that will be returned by the Arduino after a response, indicating the verification trial number, the response latency, and the pressed button(s), respectively. Finally, we add InlineRT, InlineStartTime, InlinePostSendingTime, and InlineEndTime, which are measured by our E-Prime script as a backup and control mechanism.

In addition, if feedback should be given, there should also be an attribute that lists the correct answer (we use the attribute correct for this purpose). Note that in our present setup, we only transmit integers to and from the Arduino, so the answer should be a positive integer between 0 and 32,767 (or an integer between 0 and 9 if input will be tested with the keyboard as well). Figure 1 shows an example of this table.

Setup of the stimulus presentation object In the setup of the stimulus presentation object trialStimulus, the duration is set to a short value (we used $50 \mathrm{~ms}$ ), and the PreRelease is set to the same value (entering same as duration). If feedback should be given, then Keyboard should be added as an Input Device, and the list attribute containing the correct answer will be added in the Correct field ([correct $]$ ). The Time Limit is set to same as duration. For a screenshot of these settings in E-Prime, see Fig. 2.

Note that this arrangement implies that E-Prime closes the trialStimulus object after $50 \mathrm{~ms}$, with two consequences. First, reaction time and response are set to null at this point, and have to be changed later. At the same time, these changes can only occur after that time. If reactions faster than $50 \mathrm{~ms}$ have to be measured, either the duration needs to be lower, or the script has to wait for a while.

Adding an InLine script after the stimulus presentation The trialStimulus is followed by an inline script that communicates with the Arduino. Setting the PreRelease to the same value as the duration assures that the inline script is executed immediately after the stimulus presentation is initiated. The script is shown in Listing 1 (posted as supplementary materials) ${ }^{3}$ and consists of four parts: (1) Setting up values to be transmitted, (2) Transmitting the values to the Arduino, (3) Waiting for and receiving the response from the Arduino, and (4) Disassembling the response and storing the response attributes.

As part of the setup, we fill trialNr with a random Integer value, giving each trial a unique identifier. In our example script, we send three integer values to the Arduino: (1) the trial number, which is also transmitted back by the Arduino as a control value; (2) the trial duration; and (3) a variable that could instruct the Arduino to do further action or output

\footnotetext{
$\overline{3}$ All scripts and an E-Prime template file are also available at http:// openscienceframework.org/project/cuzj5/.
} 


\begin{tabular}{|c|r|l|l|l|l|l|l|}
\hline ID & Weight & Nested & Procedure & TrialDuration & TrialNr & ArduinoTrialNr & ArduinoRT \\
\hline 1 & 20 & & itemProc & 100 & $?$ & $?$ & $?$ \\
\hline 2 & 20 & & itemProc & 100 & $?$ & $?$ & $?$ \\
\hline
\end{tabular}

\begin{tabular}{|l|l|l|l|l|l|}
\hline ArduinoStart & ArduinoButtons & InlineStartTime & InlinePostSendingTime & InlineEndTime & InlineRT \\
\hline$?$ & $?$ & $?$ & $?$ & $?$ & $?$ \\
\hline$?$ & $?$ & $?$ & $?$ & $?$ & $?$ \\
\hline
\end{tabular}

Fig. 1 E-Prime table (separated into two parts) with added fields for communication with and control of the Arduino: TrialDuration, TrialNr, ArduinoTrialNr, ArduinoRT, ArduinoButtons, InlineStartTime,

(and that we do not actually use in this example). The trial duration is read from the attribute TrialDuration set up earlier in StimulusList. Note that StimulusList is not referenced explicitly but through the Context object $c$. The three values are concatenated to one string, separated by commas, and ending with a dot. This string is written to the serial device. Two time stamps are taken, before and after doing this (see Study 5).

The script then reads continuously from the serial device, adds any string that comes in to a temporary variable, and checks whether a dot is received-which is the stop signal from the Arduino. When that has happened, another time stamp is saved to document the process, but note that this is not the actual response latency. Instead, the script expects the Arduino to send back a list of three numbers: The trial number, the response latency measured by the Arduino in microseconds, and a number coding the answer registered in the Arduino.

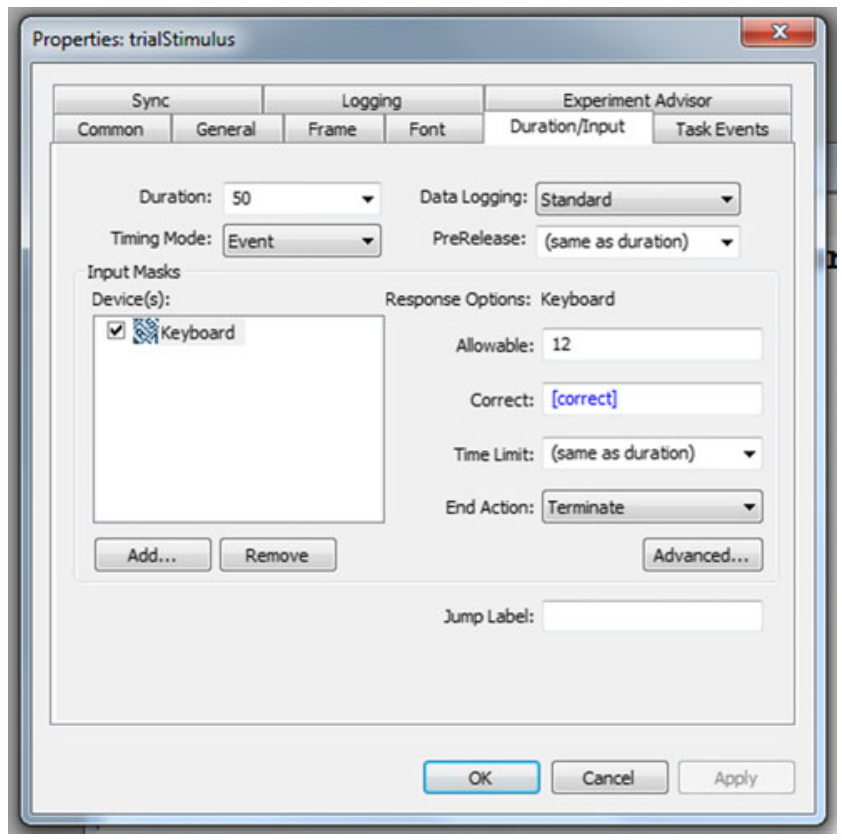

Fig. 2 Properties of the E-Prime stimulus presentation object, here a TextDisplay. The duration is set to a very short value (the actual duration is controlled by TrialDuration in the StimulusList table). Keyboard and Correct are added in order to allow feedback and testing. Data Logging is turned on
InlinePostSendingTime, InlineEndTime, and InlineRT. Only TrialDuration has to be filled in by the programming experimenter

The received string is then disassembled into these three separate numbers, which are stored in the corresponding StimulusList attributes (based on Margolis, 2011). Note that the response latency is of the type Long, which can be at maximum $2^{31}-1$. Because the unit is microseconds, the maximum response latency is thus $35 \mathrm{~min}$. The two registered time stamps (also in microseconds) are also stored, along with the difference; this difference should obviously always be larger than the response latency registered by the Arduino.

Because the trialStimulus object is already finished by the time the script receives the response, at that moment it contains only a null response, and the log will contain empty fields for reaction time and response. This is a problem if feedback needs to be provided. Thus, if an answer was registered, the script corrects the saved null response by storing the response time (but now in milliseconds to comply with E-Prime standards) and the response itself also in the attributes .RT and .RESP of the stimulus object. It also compares the response to the correct response, and adjusts .ACC accordingly. Any following FeedbackDisplay can then use trialStimulus object as its input object.

\section{Programming the Arduino to interface E-Prime}

Programs for the Arduino platform are called sketches. Our sketch (see Listing 2 in the supplementary materials) first sets up a serial connection (with the same values that are registered in Windows and E-Prime). It also sets up the pins to which the buttons are connected as input. It then waits for the transmission of three integers from E-Prime (separated by commas and ending with a dot). When these are received, it initiates a trial. At the beginning of a trial, the sketch stores a time stamp (starttime, in microseconds), and computes the maximum time by adding the read trial duration to the start time.

Using a $d o$... while loop, the sketch then continuously checks for one of the inputs to go HIGH, or for the allotted time to end. When one of these events occurs, a time stamp is taken, and used to compute the response time (which is equal or larger to the allowed trial duration if no response was given). 
The sketch then determines what should be given back as the response integer ( 0 for no response, 1 or 2 for one of the buttons, and 3 if both buttons were pressed simultaneously). Then, three integers are sent back: the trial number, the response time (in microseconds), and the response, again separated by commas and ending with a dot. This is the string that will be analyzed by E-Prime. Note that in the Arduino program, start time and end time are of the type unsigned long, which can be at most $2^{32}-1$. The clock is started when the Arduino is reset by opening the serial connection at the beginning of the experiment; the time stamps will thus turn over after about $70 \mathrm{~min}$. Experiments that run longer than that should use alternative code that only measures in milliseconds.

\section{Study 2: Checking the robustness of the communication}

In the E-Prime and Arduino code that we presented here, two safeguards are built in. First, a random number is transmitted for every trial to the Arduino, and given back by it; both are saved by E-Prime. Second, E-Prime not only stores the response latency that the Arduino measures, but also the time that passed between sending the trial to the Arduino, and receiving the answer back.

In our second test, we checked the robustness of these safeguards. Running the code described above, we repeated the test several times for different baud rates of the serial connection. Our goal was to test both whether the random number was always correctly transmitted, and how the standard deviation of the total duration was affected by serial port speed. In addition, we checked how long the Arduino waits in comparison to what it is instructed to wait.

\section{Method}

We used an Arduino Uno R3 for this test. An Atmega16U2 chip handles its USB connection. The board was connected to a Sony Laptop with Intel Core i5 processor, running EPrime 2.0.10.242. We used the scripts introduced above. For each trial, the trial duration sent to the Arduino was $100 \mathrm{~ms}$. Two buttons were connected but not pressed. ${ }^{4}$ The Arduino thus only waited for the assigned $100 \mathrm{~ms}$ (while checking the button states), and then sent back the time it actually waited. We ran 500 consecutive trials for each of the following baud rates: 14,000, 38,400, 57,600, 115,200, and 128,000 (all set in the Arduino code, E-Prime, and the Windows device manager).

\footnotetext{
${ }^{4}$ This means essentially that a pull-down resistor connected the pin to ground. Reading an unconnected pin would result in random fluctuation.
}

Results

In all 2,500 trials, the random number was correctly returned. The Arduino was instructed to wait $100 \mathrm{~ms}$. To do this, it had to check the time it already waited in a loop. In the loop, it also continuously checked the state of both buttons. Out of all trials, it reported waiting $100 \mathrm{~ms}$ in $28.0 \%$ trials, $100.004 \mathrm{~ms}$ in $38.6 \%$ of trials, $100.008 \mathrm{~ms}$ in $32.4 \%$ of trials, and 100.012 or 100.016 in $.9 \%$ of trials. (The resolution of micros() is $4 \mu$ s on $16-\mathrm{MHz}$ Arduino boards.)

To compute the delay introduced by the USB communication, we subtracted the time the Arduino reported having waited from the response latency as measured by E-primethe time between sending to and receiving from the serial port. Table 1 shows that this delay decreased steadily with increasing baud rate, from $22.73 \mathrm{~ms}$ with a baud rate of 14,400 to $5.67 \mathrm{~ms}$ with 128,000 baud. The standard deviation was also affected, dropping from 1.40 to 1.11 .

Remember that the total delay is composed of the communication to the Arduino and the communication back from the Arduino. When using Arduino as a response platform, the communication to the Arduino will be performed in addition the response latency measured by the Arduino. These data show that in order to keep the added time as short and as least variable as possible, and in order to have these measures as a backup, the highest possible baud rate should be set. Furthermore, the data also assure one that with a similar setup, the added noise would be quite low, even when factoring in both legs of communication.

In sum, this test confirms the robustness of the code in terms of accurately communicating values back and forth. Note that a similar check can be performed with any data set collected with the code above: One can always subtract the Arduino-measured RT from the inline-measured RT and check the average and variability of this delay. Finding low values for the round trip would assure one of even lower values for the first leg.

\section{Study 3: Testing the variability of the response with a photo diode}

The previous test did not actually involve any response; the Arduino simply waited for the allotted time. In a next step, we intended to test speed and variability of the script and setup with an actual measurement by the Arduino.

Method

We connected a photodiode circuit to one of the pins of an Arduino Uno R2. The circuit compared the state of a photodiode (Osram BPW 34) to the resistance by a potentiometer 
Table 1 Total delay introduced in USB communication (E-Prime-measured response latency minus Arduino-reported waiting time; all values in milliseconds, for Studies 2 and 6)

\begin{tabular}{lrrrrr}
\hline Arduino & Baud Rate & $M$ & $S D$ & Minimum & Maximum \\
\hline Uno R3 & 14,400 & 22.7323 & 1.40497 & 18.48 & 26.21 \\
& 38,499 & 10.6446 & 1.10654 & 7.23 & 12.71 \\
& 57,600 & 8.1994 & 1.07971 & 5.56 & 10.76 \\
& 115,200 & 5.7482 & 1.12930 & 3.28 & 8.37 \\
& 128,000 & 5.6715 & 1.10581 & 3.40 & 7.82 \\
Leonardo & 128,000 & 3.0997 & 0.52883 & 1.97 & 9.22 \\
\hline
\end{tabular}

using a comparator (Fairchild LM 311) and set the connected pin to HIGH or LOW, accordingly. ${ }^{5}$ The photodiode was placed at the upper half of a LCD flat screen (ASUS VE278), running at $60 \mathrm{~Hz}$, connected by HDMI to a Sony Core i5 laptop. The potentiometer was adjusted such that the pin was HIGH when the screen was white, but LOW when the screen was dark blue. The Arduino sketch was changed such that it analyzed the state of the pin connected to the photodiode circuit. Either a HIGH value or a timeout resulted in the sketch returning response time and response.

On the E-Prime side, a script presented test stimuli with either a white or a dark blue background, which always followed a black screen that was presented for $300 \mathrm{~ms}$. There were in total 500 trials with a white background and 500 trials with a dark blue background in random order. The Arduino received a waiting time of $250 \mathrm{~ms}$. Duration of the trial presentation was set to $50 \mathrm{~ms}$, and pre release to same as duration. Because the reactions from the Arduino were faster than those $50 \mathrm{~ms}$, the script also waited for an additional $100 \mathrm{~ms}$ after receiving the response to allow the trial object to finish before changing .RT and .RESP in its logs. The baud rate was set to 128,000 baud.

\section{Results}

In all of the 1,000 trials, the photodiode correctly identified the screen as white or dark blue. One trial however showed an outlier low response time of $12 \mu \mathrm{s}$ (for a white trial), probably due to volatility in the photo diode, and was removed from the analysis. The data for the blue trials resemble Study 2, and we do not report them here.

For the white trials, the response time reported by the Arduino ranged from 5.43 to $6.68 \mathrm{~ms}, M=6.031 \mathrm{~ms}, S D=$ $0.117 \mathrm{~ms}$. We again computed a delay as the difference between the response time measured by the E-Prime script (including both legs of communication) and the Arduinomeasured response time. The delay varied between $3.12 \mathrm{~ms}$ and $10.43 \mathrm{~ms}, M=5.350, S D=1.176 \mathrm{~ms}$.

Assuming the screen had a perfect constant switch time from black to white, these data show that when using the full

\footnotetext{
$\overline{{ }^{5} \text { See https://reactiontimes.wordpress.com/electro-mechanical-turk/ for }}$ the layout and further details.
}

potential of the Arduino, it is possible to measure these switching times with a standard deviation of little more than a tenth of a millisecond. Even if the timing is left to E-Prime itself, we still only get noise with a $S D$ of about $1 \mathrm{~ms}$.

One might think that the $S D=0.117 \mathrm{~ms}$ overestimates the precision because the communication from E-Prime to the Arduino has an additional variability that is not captured here. However, note that to then explain the low variability of the times measured by the Arduino, one would have to assume that the true switching of the screen counteracted the variability in the communication, which is very unlikely. Thus, the tenth of a millisecond is likely close to the true precision.

\section{Study 4: Comparing an Arduino response box to other response boxes}

Test 3 was instructive concerning the absolute performance of the Arduino platform; however, a comparison to commercial response boxes would be interesting. In Study 4, we thus compared the accuracy of a response box built using the Arduino to a standard serial response box and a keyboard, always using E-Prime.

\section{Method}

To improvise an Arduino response box, we connected two Cherry MX key modules to the Arduino in the standard way (http://arduino.cc/en/Tutorial/Button), using an Arduinocompatible rapid prototyping kit (Seeed Studio Grove Base Shield and its screw terminal connector). The Arduino sketch was adapted such that it continuously read the states of both input pins and the timeout. The key modules were mounted on Lego Duplo bricks for a solid foundation (see Fig. 3).

As a comparison, we used a PST serial response box connected via a serial port to a desktop PC running Windows 7 and E-Prime 2.0. We also compared the performance of a standard USB Microsoft keyboard.

We created an E-Prime experiment that displayed 100 very simple trials: In each trial, a black waiting screen was 


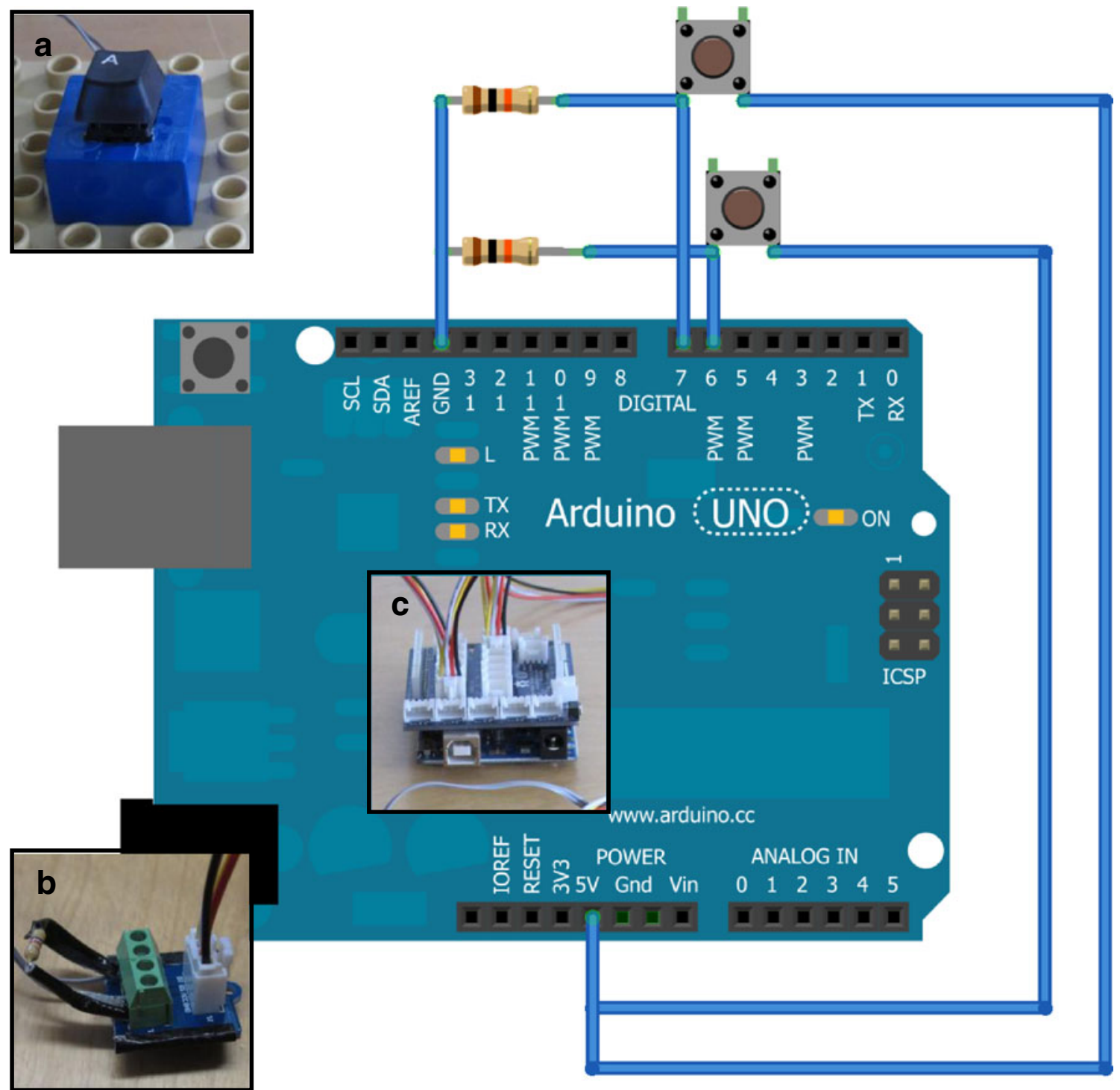

Fig. 3 Connecting two buttons to the pins 6 and 7 of an Arduino, realized with Cherry key modules (inset a), screw terminals (inset b), and a rapid prototyping base shield (inset $\mathbf{c}$ )

followed by a white target screen. The program then waited for a button on the response device to be pressed. To press the button in response to the white screen onset, we constructed a "robot," using a second Arduino that was not connected to the computer itself. This Arduino featured the photodiode circuit also used in Study 3, placed on the screen (Asus VG236H set to $60 \mathrm{~Hz}$ ). In addition, it was connected to a $12-\mathrm{V}$ push solenoid (Intertec ITS-lz-2560 d$12 \mathrm{vdc})$. This solenoid was placed above the button of the response box, pushing it when turned on. The sketch running on this second Arduino continuously checked whether the photodiode pin was set HIGH or LOW; when it was $\mathrm{HIGH}$, the solenoid was fired, which pressed the button and triggered the end of the trial, releasing the solenoid. The code reading the photodiode was adapted such that a change in brightness was only registered if two continuous readings resulted in the same value, to make the program more robust (see note 5).

Results

We ran two tests of 100 items with each hardware; we report both separately to convey the reliability of the measurement. Because we compared the Arduino measurement to the EPrime measurement for the two devices, the Arduino also only measured in milliseconds.

The crucial numbers in this test were the standard deviations. The total reaction times should be interpreted with caution, because they were based on completely different hardware, and it is possible that the solenoid hit the different 
buttons in slightly different ways, with different travel distances, and so forth.

The standard in this test was set by the serial response box, usually regarded as millisecond-precise. Table 2 indeed shows two standard deviations between 1 and $1.4 \mathrm{~ms}$; the range of responses is impressively low. These small numbers confirm that our Arduino-solenoid-robot also performed quite reliably - at least with the precision found here. The overall latency was rather high, between 56 and $58 \mathrm{~ms}$. We assume that this was due to the large travel of the solenoid and the specifics of the circuit.

Our Arduino response platform performed almost as well as the PST serial response box, with two SDs of 1.28 and $1.29 \mathrm{~ms}$. This shows that the combination of PC-to-Arduino USB communication and having the Arduino measuring the RT makes up for the disadvantage of having no real direct serial connection to the PC.

The keyboard performed worse than the two other devices, but still with an acceptable noise $S D$ of about $3 \mathrm{~ms}$. Note, however, that this cannot be generalized to USB keyboards in general, and hardware details can vary even within one product line.

To compare the variance in the various tests, we computed Levene's tests for equality of variances. Regarding their variances, the two Arduino measures did not differ from each other, and neither did the two keyboard measures, both $F_{\mathrm{s}}<1$. However, the variances of the two response box measures did differ, $F(1,198)=4.63, p=.033 .{ }^{6}$ Importantly, the two Arduino measure variances did not differ from the two response box variances, $F_{\mathrm{S}}(1,198)<1.81, p \mathrm{~s}>.180$. The keyboard variances differed from all other variances, $F \mathrm{~s}(1,198)>51, p \mathrm{~s}<.001$. Figure 4 shows the distributions. In sum, both serial response box and Arduino performed much better than a keyboard; and the difference between Arduino and response box seem to be not larger than the difference between two different tests with the response box.

\section{Study 5: Estimating the delay from trial initiation in E-Prime to start of clock in Arduino}

In the script developed here, we send a signal from E-Prime to the Arduino at the start of a trial. This signal prompts the Arduino to start its time measurement. Obviously, the time that the signal takes to travel from E-Prime to the Arduino is not included in the final response latency reported back by the Arduino. In Study 1, we found that the average of this additional delay was $1.25 \mathrm{~ms}$, with a very low $S D$. Nevertheless,

\footnotetext{
$\overline{6}$ The difference between the two tests for the response box is surprising but remains unclear. We ran two more tests with 100 trials each, but the results resemble those of the run with the larger variance.
}

Table 2 Latencies of responses (in milliseconds) by the "robot" (second Arduino equipped with photodiode and solenoid) reacting to the onset of a white screen (Study 4)

\begin{tabular}{lccccc}
\hline Hardware & Test & $S D$ & $M$ & Min & Max \\
\hline PST serial response box & 1 & 1.08 & 56.28 & 54 & 59 \\
& 2 & 1.34 & 57.54 & 54 & 61 \\
Arduino & 1 & 1.29 & 42.38 & 38 & 45 \\
& 2 & 1.28 & 41.87 & 40 & 46 \\
Microsoft USB keyboard & 1 & 2.99 & 69.67 & 63 & 77 \\
& 2 & 3.09 & 72.25 & 65 & 79 \\
\hline
\end{tabular}

ignoring this delay would reduce the accuracy of the measurement and might be undesirable in some contexts.

Above, we mentioned that the E-Prime scripts stores two time stamps, one immediately before sending the signal, and another one upon receiving the data back from the Arduino. By testing how much longer this difference is than the latency reported by the Arduino, researchers can judge the reliability of the USB speed, but this delay will include both legs of communication, not the first delay in particular.

This problem was already tackled by Li et al. (2010) in the development of their RTbox. Their software similarly sends a start trial signal to an external microprocessor and records time stamps right before and after that operation. In addition, their microprocessor software takes and reports a time stamp upon receiving the trial start signal. This differs slightly from our approach so far, because on the E-Prime side, we analyzed only the time stamp before sending a signal, and on the microprocessor side, we reported back only the time difference.

Li et al. (2010) discussed three different methods for estimating the true moment of sending the signal from computer to microprocessor. Each relies on taking several samples (at least 20). One possibility is to compute the difference of the two computer time stamps before and after sending, and to select the shortest one. This has to include the true time of sending the signal, and to estimate that moment, Li et al. simply averaged the two time stamps associated with that shortest delay.

In another method, they computed the difference between the computer time stamp before sending and the microprocessor time stamp upon receiving. Note that these will have an unknown offset because the clocks of the two processors are started at different times. Li et al. (2010) then selected the minimum difference of the two time stamps and used the associated computer time stamp before sending as an estimate of the true moment of sending. ${ }^{7}$

\footnotetext{
${ }^{7}$ A third method, which is shown to be slightly inferior, uses the difference between microprocessor time stamp and computer time stamp after sending, but we will not discuss this here further.
} 
Fig. 4 Histograms of measurements in Study 4. For each periphery, two measures with 100 trials each were taken. $\mathrm{SRB}=$ serial response box, $\mathrm{ARD}=$ Arduino, $\mathrm{KB}=$ keyboard

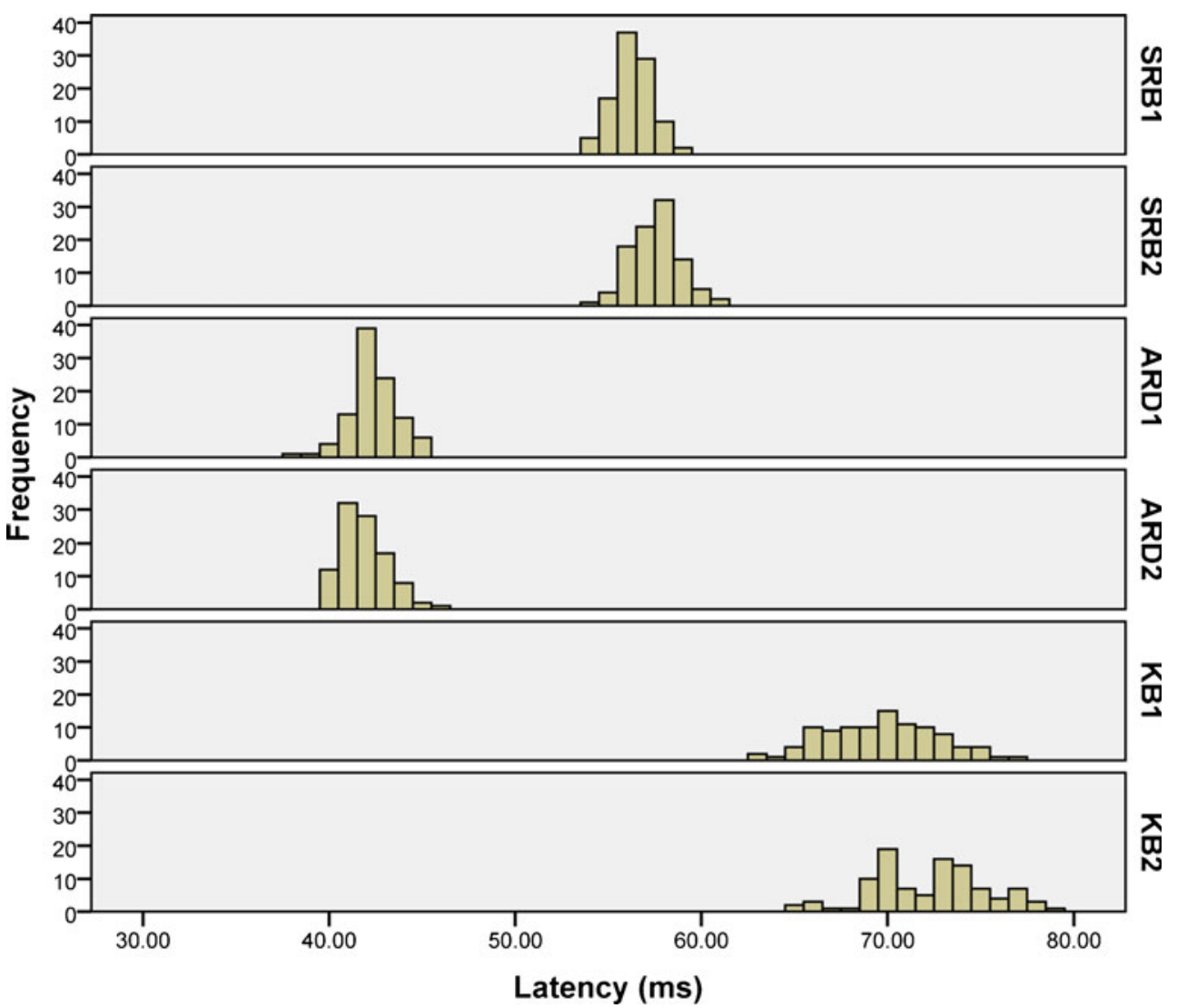

In order to demonstrate how the methods devised by Li et al. (2010) can be applied in our scripts as well, we ran another study in which we could record time stamps both before and after sending the serial signal, in order to investigate the differences between these time stamps and their distributions.

\section{Method}

On the Arduino side, we changed the script such that the time stamp of starting the trial is also returned, in addition to the latency. On the E-Prime side, we stored this additional time stamp from the Arduino. We used an Arduino Leonardo, connected with 128,000 baud to E-Prime 2.0.10.242 running on a Windows 7 laptop.

We ran consecutive 300 trials. In each, E-Prime initiated a trial with 100-ms duration, saving time stamps before and after sending serial communication. The Arduino only waited the allotted time without any further measurement. In E-Prime, before each trial we added a random delay between 1 and $100 \mathrm{~ms}$, in order to desynchronize the process from the computer's USB cycles, as would be the case in a normal experiment. Additional constant delays were present as well. The complete test took about $12 \mathrm{~min}$.

\section{Results}

We first checked the delay between the time stamps taken by E-Prime before and after sending the serial communication. The average delay was $0.341 \mathrm{~ms}$, ranging from 0.16 to 0.55 $(S D=0.087)$. Following Li et al., we selected the minimum delay and used the average of the two associated E-Primerecorded time stamps (before and after) to estimate the true sending time. In our experiment, this arrived at an offset of $777,411.246 \mu$ s between the two clocks (due to unequal starting times of the experiment on computer vs. Arduino).

We then investigated the difference between the E-Prime time stamp before sending, and the Arduino time stamp upon receiving the start trial signal. The average of this delay is uninformative because they were taken with two different, unsynchronized clocks. The $S D$ was rather large, at $7.926 \mathrm{~ms}$. However, note that these 300 measurements were taken over the course of $12 \mathrm{~min}$. Li et al. (2010) showed that, due to small differences in the actual speed of clocks, drifts between them occur that have to be removed. When we regressed the differences on the number of trial, we found a large linear effect of trial number, $\beta=$ $.999, t(299)=495, p<.001$. After removing this linear influence, the unstandardized residuals had a much lower $S D$ of $0.28 \mathrm{~ms}$. Again following Li et al., when using the 
sending time associated with the shortest difference residual, we arrived at an offset of $777,411.325 \mu \mathrm{s}$, almost identical to the result from the first method.

\section{Discussion}

In the scripts above, we ignored the communication delay between sending the trial start signal from E-Prime and receiving it in the Arduino. However, if concern arises or the paradigm requires it, the true time of sending, and thus the true response latency including the communication delay, can be estimated following the methods devised by Li et al. (2010). For this purpose, the two time stamps taken by EPrime before and after sending can be used effectively. Essentially, this approach synchronizes the clock in the Arduino and the clock in the computer, which are always started at different times with an unknown offset.

Li et al. (2010) actually proposed the synchronization of clocks in this manner before each trial, implementing between 20 and 100 communication cycles between computer and microcontroller in order to estimate the true RT. Note that while this increases accuracy of the latency measurement in terms of getting closer to the true value, it does not increase precision, because the measurement itself is again a new random sample from the distribution identified for the duration of communication.

Given that all of our results (and theirs) show that this delay is shorter than $1 \mathrm{~ms}$, we do not believe that this is necessary for most purposes. However, we definitely recommend checking both (a) the duration of sending the serial communication and (b) the difference between Arduino-reported latency and total E-Prime measured latency for all trials to assure against general malfunctioning and sudden drops in performance.

As we already noted, this approach only estimates the delay between start of a trial and start of time measurement in the Arduino. To get an accurate measurement instead of an estimate, it would be necessary to program the Arduino such that it detects the onset of a trial itself. This could be implemented by signaling the onset of a trial with a cue on the screen (e.g., a dot in a corner), and equip the Arduino with a light sensor that allows it to save a time stamp at the true onset. Although we did not implement this for the present article, it could be easily done. Together with Study 3 , the following study will provide a background for the precision of such an approach.

\section{Study 6: Emulating human interface devices with the Arduino Leonardo}

In Study 1, we determined that communication from the computer to the Arduino board was faster and less variable than communication from the Arduino back to the computer. We therefore developed scripts that used only the first direction under time critical conditions, and then let the Arduino measure the response latency and report it back when timing was not critical anymore. This allowed the Arduino also to receive instructions for stimulus presentation. The cost was that we needed to add special scripts to the latency-collecting software.

In our Study 6, we also wanted to explore how an Arduino response box can be constructed without relying on specialized scripts. For this purpose, we took advantage of the recently introduced Arduino Leonardo. This board does not feature a separate chip that handles communication with the USB. Instead, the main processor, an Atmel ATmega32u4, also handles this communication. In all other respects, this board is similar to the Arduino Uno R3.

To estimate the performance of the Leonardo, we first repeated the fastest condition of Study 2 (128,000 baud) with the Leonardo board. We encountered a higher percentage of longer waiting times, but note this concerned only microsecond differences: The program was scheduled to wait only $100 \mathrm{~ms}$; it reported waiting exactly that long in $25 \%$ of trials. On about half of the trials (47.2\%), it waited $100.004 \mathrm{~ms}$; on a fourth of the trials $(26.4 \%$ ), it waited $100.012 \mathrm{~ms}$; and on $1.4 \%$, it waited 100.016 or $100.020 \mathrm{~ms}$. The last line of Table 1 shows the delay, computed as the difference between the time of the total round trip measured by E-Prime and the time waited by the Arduino. This delay had an average of $3.10 \mathrm{~ms}$ with an $S D$ of $0.53 \mathrm{~ms}$, ranging from 1.97 to $9.22 \mathrm{~ms}$. These values indicate that the distribution is quite narrow with only a few outliers. Indeed, $99.4 \%$ of the delays were below $3.72 \mathrm{~ms}$, and there were three outlier values between 7.2 and $9.23 \mathrm{~ms}$. This suggests that the Leonardo could be a viable alternative to the Uno despite the fact that the main processor handles also USB communication. At least with simple tasks like in this test (checking the status of two input pins), USB communication seemed to be even faster than with an Uno, although there were $0.6 \%$ outliers with higher delays.

However, the main advantage of the Leonardo is another feature. Running USB connection in the main processor allows the Arduino to emulate a keyboard or a mouse when connected by USB. In other words, the Leonardo can be programmed to simulate the press of a keyboard button in response to events, and response latency software will recognize this buttonpress as if it came from a regular keyboard. Of course, this signal still has to go through the regular polling process of the operating system, but there is a chance that noise coming from the construction of a regular keyboard will be avoided in this way (Li et al., 2010).

\section{Method}

To evaluate this option, we connected an Arduino Leonardo to a light-dependent resistor (LDR), in the form of the 
TinkerKit LDR Sensor sold by Arduino.cc. We intentionally chose this module to demonstrate accuracy using standard commercial components, and to our best knowledge there is no photo diode module for any of the Arduino rapid prototyping platforms. The LDR was placed in the right top corner of the screen of a Sony Vaio laptop. We programmed the Arduino such that it would continuously read the state of the LDR, and simulate the pressing of key A when the measurement surpassed the level 200.

Note that the LDR was connected to an analog pin on the Arduino. Reading such a pin takes longer than reading a digital pin, about $0.1 \mathrm{~ms}$. We added a further delay of 10 microseconds to allow complete settling of the analog to digital converter. As a result, all internal reading on the Arduino should have values multiple of approximately $0.11 \mathrm{~ms}$.

On the Windows side, we programmed a simple experiment in both E-Prime 2.0.10.242 and DMDX 4. In both cases, there were 200 trials. Each trial first showed a black screen for 2,000 ms, and then a white screen, waiting for the pressing of the button A. The keypress was measured with the regular functions in E-Prime and DMDX.

As a comparison, we added one condition in which we used the script developed above (E-Prime only because DMDX does not support a serial interface), thereby measuring response latency on the Arduino directly. In this case, the Leonardo was connected with a speed of 128,000 baud, and the script checked the state of the LDR.

\section{Results and discussion}

Note that E-Prime usually reports measurements in milliseconds, whereas our E-Prime script and DMDX report in microseconds. When the Leonardo was programmed to return the letter A upon the screen turning white, DMDX recorded latencies between 5.53 and $6.69 \mathrm{~ms}, M=6.09, S D=$ 0.31 (condition DMDX/Emulation). E-Prime reported latencies between 6 and $9 \mathrm{~ms}, M=7.31, S D=0.52$ (condition EPrime/Emulation). When E-Prime and Leonardo were programmed with the scripts developed in the present article (Study 2), the latencies ranged from 4.10 to $5.26 \mathrm{~ms}, M=4.65$, $S D=0.29$ (condition Arduino measurement). We compared the variances and means with Levene's tests and $t$ tests, respectively. DMDX/emulation and Arduino measurement did not differ from each other regarding variances, $F<1$, but regarding means, $t(398)=47.78, p<.001$. EPrime/emulation differed from both other conditions regarding variances, $F_{\mathrm{s}}(398)>94, p \mathrm{~s}<.001$ and means, $t \mathrm{~s}>28, p \mathrm{~s}<.001$.

These tests show remarkable accuracy for the Arduino Leonardo, even when it is used simply to emulate a keyboard device. We achieved $S D$ s of half a millisecond in both E-Prime and DMDX. DMDX even rivaled the script developed above, as the $S D$ of its measurements was not significantly higher than the measurements run on the Arduino itself. E-Prime performs less well, perhaps simply because it measures only in milliseconds, but its performance is still very good. This suggests that if the Arduino is not used to present other stimuli triggered by the communication it receives from E-Prime, using an Arduino Leonardo and emulating a HID device might be sufficiently accurate.

In addition, together with Study 3, these results suggest that adding a light sensor can be a valid approach to allow the Arduino registering the onset of a trial, and thereby increase accuracy of measurement.

\section{General discussion}

Current response time measurement in cognitive science relies heavily on measuring latencies of key presses on standard keyboards. Although our Study 4 showed that the precision of such measurements could be acceptable, this hinges immensely on the particular keyboard and paradigm. Furthermore, key presses might not always be the ideal movement to measure. More precise response boxes are expensive and often rely on legacy interfaces or on specific software. Instruments that measure other movements are even more expensive and difficult to program.

In the present article, we described a way out of this dilemma. We show how the open source microcontroller platform Arduino can be combined with a standard software package, E-Prime, to measure response latencies as precisely as a serial response box does. We show that flexible millisecond precision is within the reach with hardware investments of less than $€ 50$.

In Study 1, we saw that communication in the direction from the PC to the Arduino is faster and less noisy than the return way. We therefore program the Arduino such that it gets signaled when a trial starts, measures the response latency itself, and then reports back both the latency and the response. We developed an E-Prime inline script that signals the trial to the Arduino, waits for the measured values, and saves them to the database. Study 2 showed that this communication is reliable and fast when high baud rates are selected. Study 3 confirmed that with this script, the setup of PC, Arduino, and E-Prime can measure an event (here a change on the screen) with excellent precision (an $S D$ of $<1 \mathrm{~ms}$ ). Study 4 confirmed that a response box built with this setup performs as well as a serial response box, the current gold standard, and better than a keyboard. Study 5 showed that by taking additional time stamps into account, accuracy can be enhanced. Finally, Study 6 demonstrated that even without using special scripts, the Arduino Leonardo board could be used to construct a millisecond precise response box that can be used with any software. 
Our script includes two quality assurance mechanisms: a check of the correct match of E-Prime and Arduino trial via a random number, and a backup measurement of the latency via the script. Both should be used to verify the correct implementation of the script (see a check list in the Appendix, posted as supplementary materials with the article).

We focused here on replicating response box and keyboard functionality because this is the current norm. However, the Arduino has potential for building new kinds of response measures. It can be connected to a multitude of sensors to develop new paradigms in an easy fashion. Rapid prototyping toolkits offer gyroscopes, accelerometers, lightdependent resistors, hall sensors, compasses, touch sensors, and much more. Here we only demonstrate the precision using keys (Study 4), photodiode (Study 3), and lightdependent resistors (Study 6). Tests with other hardware have to be conducted in the future.

Interestingly, the script that we developed here can be directly used to also implement the presentation of stimuli via the Arduino. For instance, in some paradigms vibration or LEDs have to be used (e.g., Pavani \& Castiello, 2004). LEDs and small vibrators can easily be connected to an Arduino, and the Arduino can control them in response to the trial signal. We believe that controlling external actuator and measurement hardware from experimental software like E-Prime via an Arduino can greatly enhance the experimental psychologist's toolbox.

\section{Caveats and future directions}

Perhaps the biggest problem for anybody who wants to implement our solution right away is that in rapid prototyping kits for the Arduino, there seems to be shortage of good button modules. While this situation remains, we suggest three solutions. Most easily, any button can be connected to an Arduino directly. ${ }^{8}$ We recommend employing Cherry key modules.

Second, it is clear that our E-Prime InLine script requires basic knowledge in scripting, and is not as easily deployed as simply choosing an input device in E-Prime. However, there are very few changes that experimenters need to make in a standard experiment. We are currently working on packaging the script such that it is more easily employed.

Third, we are certainly aware that other software packages exist that also implement serial ports (e.g., Presentation). We expect that our script can be easily ported to these packages. We make our script available under the GNU GPL v3 public license and encourage porting to other environments.

We focused here on standard Arduino USB communication, but we would like to point out that alternatives are

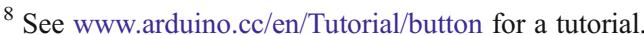

available. For instance, it is conceivable to use parallel ports where they are still available to communicate with an Arduino, or to employ a DAQ card. Another option is to circumvent the hardware that an Arduino offers for USB connection, because this is not optimized for extreme speeds. For instance, it is possible to connect the hardware serial port of an Arduino board to a special FTDI chip, which then talks to the computer via USB and specialized drivers. In initial tests we have found an improved accuracy using this setup.

Finally, we want to reiterate that despite its many advantages, the use of additional hardware may not be necessary in some paradigms. Studies on response latencies have been successfully conducted over the Internet, which presents the most noisy environments imaginable, with many different hardware configurations (Nosek, Banaji, \& Greenwald, 2002). The challenge is to judge a priori, on the basis of expected effect size, desired test power, and response latency distribution, what noise induced by the particular hardware will be acceptable. Providing guidelines for this remains a task for the future.

We hope that experimental psychologists increasingly abandon the keyboard as a response device when it is not the ideal index of cognition. For many researchers in cognitive science, programming their own paradigms has become an elementary part of developing studies. We believe that building suitable hardware can become similarly useful and natural.

Author Note The main part of this research was conducted at the Instituto Universitário de Lisboa (ISCTE-IUL), Centro de Investigação e Intervenção Social, Avenida das Forças Armadas, 1649-026 Lisbon, Portugal, with support from Fundação para a Ciência e a Tecnologia (FCT), Grant No. PTDC/PSI-PSO/101366/2008. T.W.S. is now at the University of Oslo. We thank Volker Seibt, Robert Richards, Gonçalo Paiva, Pedro Antunes, and João Fonseca for their help with hardware, and Carla Murteira, Elizabeth Collins, and Diniz Lopes for their help and comments on this project.

\section{Appendix}

Checklist before collecting data

1. Is the same highest possible baud rate set in E-prime, Arduino sketch, and device manager?

2. Are all necessary columns added to the list containing the stimulus object or a higher order list?

3. Are the names of the stimulus object used in the final part of the E-Prime InLine adjusted to names actually used?

4. Does the E-Prime script leave a grace period of ca. $2 \mathrm{~s}$ after the start, before the first communication with the Arduino is attempted?

5. Is data logging turned on for the stimulus object? 
6. Does the Arduino script define the correct pins for input and ouput?

Checklist after collecting data

7. Is the TrialNr (which is sent to the Arduino) the same as ArduinoTrialNr (which is received from the Arduino) for all trials?

8. Compute the difference inlineRT - arduinoRT, and check its distribution. If the average is large or the standard deviation is high, ArduinoRTs are going to be inaccurate or imprecise, respectively, as about $1 / 3$ of the delay happens before ArduinoRT is measured.

9. Compute the difference InlinePostSendingTime InlineStartTime. Trials with outlier differences may be unreliable, as the trial onset stimulus might have been delayed.

\section{References}

Brainard, D. H. (1997). The psychophysics toolbox. Spatial Vision, 10, 433-436. doi:10.1163/156856897X00357

Canto, R., Bufalari, I., \& D'Ausilio, A. (2011). A convenient and accurate parallel Input/Output USB device for E-Prime. Behavior Research Methods, 43, 292-296. doi:10.3758/s13428010-0022-3

D'Ausilio, A. (2012). Arduino: A low-cost multipurpose lab equipment. Behavior Research Methods, 44, 305-313. doi:10.3758/ s13428-011-0163-z

Forster, J. C. (2012). DMDX Help Input. Retrieved August 16, 2012 , from www.u.arizona.edu/ jforster/dmdx/help/dmdxhinput.htm
Forster, K. I., \& Forster, J. C. (2003). DMDX: A Windows display program with millisecond accuracy. Behavior Research Methods, Instruments, \& Computers, 35, 116-124. doi:10.3758/BF03195503

Galilei, G. (1638). Discorsi e dimostrazioni matematiche, intorno à due nuove scienze. Leiden, The Netherlands: Lodewijk Elzevir.

Harmon-Jones, E. (2011). How to make a reaction time box for $D M D X$. Retrieved November 20, 2011, from www. socialemotiveneuroscience.org/making_RT_box_DMDX.pdf

Li, X., Liang, Z., Kleiner, M., \& Lu, Z.-L. (2010). RTbox: A device for highly accurate response time measurements. Behavior Research Methods, 42, 212-225. doi:10.3758/BRM.42.1.212

Margolis, M. (2011). Arduino cookbook. Sebastopol: O'Reilly Media.

Nosek, B. A., Banaji, M., \& Greenwald, A. G. (2002). Harvesting implicit group attitudes and beliefs from a demonstration web site. Group Dynamics: Theory, Research, and Practice, 6, 101-115. doi:10.1037/1089-2699.6.1.101

Pavani, F., \& Castiello, U. (2004). Binding personal and extrapersonal space through body shadows. Nature Neuroscience, 7, 14-16. doi:10.1038/nn1167

Plant, R. R., Hammond, N., \& Turner, G. (2004). Self-validating presentation and response timing in cognitive paradigms: How and why? Behavior Research Methods, Instruments, \& Computers, 36, 291-303. doi:10.3758/BF03195575

Plant, R. R., Hammond, N., \& Whitehouse, T. (2003). How choice of mouse may affect response timing in psychological studies. Behavior Research Methods, Instruments, \& Computers, 35, 276284. doi:10.3758/BF03202553

Plant, R. R., \& Turner, G. (2009). Millisecond precision psychological research in a world of commodity computers: New hardware, new problems? Behavior Research Methods, 41, 598-614. doi:10.3758/BRM.41.3.598

Voss, A., Leonhart, R., \& Stahl, C. (2007). How to make your own response boxes: A step-by-step guide for the construction of reliable and inexpensive parallel-port response pads from computer mice. Behavior Research Methods, Instruments, \& Computers, 39, 797-801. doi:10.3758/BF03192971 